\title{
Diabetes insipidus
}

National Diabetes Information Clearinghouse (NDIC)

\section{Definitions}

Blood glucose level

Defined by National Diabetes Information Clearinghouse (NDIC)

Kidney

Defined by National Diabetes Information Clearinghouse (NDIC)

Source

National Diabetes Information Clearinghouse (U.S.). (2009). The diabetes dictionary. [Bethesda, Md.]: U.S. Dept. of Health and Human Services, National Institutes of Health, National Institute of Diabetes and Digestive and Kidney Diseases, National Diabetes Information Clearinghouse.

A condition characterized by frequent and heavy urination, excessive thirst, and an overall feeling of weakness. This condition may be caused by a defect in the pituitary gland or the kidney. In diabetes insipidus, blood glucose levels are normal. 